\title{
MicroRNA-218 targets adiponectin receptor 2 to regulate adiponectin signaling
}

\author{
HECHUN DU ${ }^{1}$, ZIMU FU ${ }^{1}$, GUOHUA HE $^{1}$, YUEJUAN WANG ${ }^{1}$, GUIYU XIA ${ }^{1}$, MIN FANG ${ }^{1}$ and TAO ZHANG $^{1,2}$ \\ ${ }^{1}$ Shaoxing Women and Children's Hospital, Shaoxing, Zhejiang 312000; ${ }^{2}$ Women's Hospital, \\ School of Medicine, Zhejiang University, Hangzhou 310058, P.R. China
}

Received April 14, 2014; Accepted January 7, 2015

DOI: $10.3892 / \mathrm{mmr} .2015 .3282$

\begin{abstract}
Adiponectin exerts an antidiabetic function through the adiponectin receptors 1 and 2 (AdipoR1 and AdipoR2). The mechanism regulating the expression of adiponectin receptors remains to be elucidated. Bioinformatics analysis demonstrated that microRNA (miR)-218 targets the 3' untranslated region (3'UTR) of the AdipoR 2 mRNA. The present study aimed to investigate whether miR-218 regulated the expression of AdipoR2 using immunoblotting, reverse transcription quantitative polymerase chain reaction and luciferase assays. The protein level and the mRNA level of AdipoR2 were reduced when miR-218 was expressed in HepG2 cells. Additionally, overexpression of miR-218 repressed the activity of a luciferase reporter containing the 3'UTR of AdipoR2. Furthermore, the present study aimed to determine whether miR-218 regulated glucose metabolism through detecting signaling pathways and glucose uptake. The phosphorylation of AMP-activated protein kinase and p38 mitogen-activated protein kinase was reduced in miR-218-expressing cells. In addition, miR-218 inhibited adiponectin-induced glucose uptake. The present results suggested that miR-218 targets AdipoR2 to inhibit adiponectin signaling.
\end{abstract}

\section{Introduction}

Adiponectin, derived from adipocytes, is a circulating plasma protein. It has been observed to have a number of effects on

Correspondence to: Dr Tao Zhang or Mr. Min Fang, Shaoxing Women and Children's Hospital, 305 East Street, Shaoxing, Zhejiang 312000, P.R. China

E-mail: epitach123@gmail.com

E-mail: fangming0005@sina.com

Abbreviations: MAPK, mitogen-activated protein kinase; AdipoR, adiponectin receptor; AMPK, AMP-activated protein kinase; PPAR, peroxisome proliferator-activated receptor

Key words: adiponectin, adiponectin receptor 2, microRNA-218, AMP-activated protein kinase, p38 mitogen-activated protein kinase metabolism, including insulin sensitization and vascular protective properties (1). Human studies have revealed that in obese individuals plasma adiponectin levels are significantly decreased (2), as is the case in patients with insulin resistance (3) and Type 2 diabetes (4). Insulin resistance may be induced in mice via targeted deletion of the adiponectin gene (5), whereas increasing the expression of adiponectin resulted in a greater level of insulin sensitivity $(3,6)$. Such findings have led to the hypothesis that adiponectin is an anti-inflammatory, insulin-sensitizing adipokine, which may have protective effects in metabolic diseases associated with obesity.

The adiponectin receptors 1 and 2 (AdipoR1 and AdipoR2) mediate the physiological effects of adiponectin in the liver and in the skeletal muscle, regarding the metabolism of glucose and lipids (7). The adiponectin receptors are 7-transmembrane proteins. The signaling pathways responsible for the effects of adiponectin on metabolism remain to be fully elucidated. The adiponectin-activated AMP-activated protein kinase (AMPK), peroxisome proliferator-activated receptor (PPAR)- $\alpha$, and the p38 mitogen-activated protein kinase (MAPK) pathways (7) have all been revealed to involve AdipoR1 and AdipoR2, and to mediate the metabolism of glucose and lipids. However, the mechanism, which regulates the expression of the adiponectin receptors remains to be elucidated.

MicroRNAs (miRNAs) are a class of 22 nucleotide noncoding RNAs, which regulate gene expression at the post-transcriptional level. miRNAs silence their target genes by cleaving mRNA molecules or inhibiting their translation and thus regulate various physiological and pathological processes (8). A previous study indicated that miRNAs act as key regulators in insulin signaling and glucose metabolism (9). For example, miR-375 was observed to suppress glucose-induced insulin secretion (10). Other miRNAs, including Let-7 $(11,12)$, miR-103, miR-107 (13) and miR-29a/b (14) were revealed to regulate insulin sensitivity in the liver and peripheral tissues by controlling the expression of numerous components of the insulin signaling pathway. In the present study, it was observed that miR-218 inhibits adiponectin signaling and glucose uptake by targeting AdipoR2.

\section{Materials and methods}

Cell culture. HepG2 cells (American Type Culture Collection, Manassas, VA, USA) were maintained in Dulbecco's 
modified Eagle's medium (DMEM; Invitrogen Life Technologies, Carlsbad, CA, USA) supplemented with $10 \%$ fetal bovine serum (Hyclone, Waltham, MA, USA) at $37^{\circ} \mathrm{C}$.

Reverse transcription quantitative polymerase chain reaction $(R T-q P C R)$. Total RNA was isolated using TRIzol reagent (Invitrogen Life Technologies) according to the manufacturer's instructions. Equal amount of total RNA $(1 \mu \mathrm{g})$ were reverse transcribed using a High-Capacity cDNA Reverse Transcription kit (Applied Biosystems, Foster City, CA, USA). qPCR analysis was performed in $10 \mu \mathrm{l}$ reactions using the ABI7900 Real-Time PCR system (Applied Biosystems) and SYBR Green PCR Master Mix (Applied Biosystems). The cycling conditions were as follows: Initial denaturation at $95^{\circ} \mathrm{C}$ for $30 \mathrm{sec}$, then 40 cycles of denaturation at $95^{\circ} \mathrm{C}$ for $10 \mathrm{sec}$ and annealing and extension at $60^{\circ} \mathrm{C}$ for $30 \mathrm{sec}$. The following primers (Sangon Biotech Co., Ltd., Shanghai, China) were used: AdipoR2 forward, 5'-CCCAAGAAGTCCGAGACACG-3' and reverse, 5'-TGTTGGCTCGTTCATGGGAT-3'; and $\beta$-actin forward, 5'-TTGCGTTACACCCTTTCTTG-3' and reverse, 5'-CACCTTCACCGTTCCAGTTT-3'. Expression of AdipoR2 was normalized relative to $\beta$-actin using the $2^{-\Delta \Delta C T}$ method.

Plasmid construction. The wild-type 3'-untranslated region (3'-UTR) of the AdipoR2 gene containing the predicted miR-218 target sites was amplified using polymerase chain reaction (PCR) from HepG2 cDNA using the following primer set (Sangon Biotech Co., Ltd.): AdipoR2, forward 5'-GCTCTAGATACCTACCAGTCTCCAGG-3' and reverse 5'-GCTCTAGATAGAAACTGAGAATACAGC-3'. The 3'-UTR fragment was cloned downstream of the firefly luciferase coding region in the XbaI site of the pGL3-control plasmid (Promega Corporation, Madison, WI, USA).

Transfection. HepG2 cells were transiently transfected with $50 \mathrm{nM}$ of the chemically synthesized miR-218, 5'-UUGUGCUUGAUCUAACCAUGU-3', or negative control miRNA, 5'-UUCUCCGAACGUGUCACGU-3' (Genepharma, Shanghai, China), using Lipofectamine 2000 (Invitrogen Life Technologies) according to the manufacturer's instructions. After $24 \mathrm{~h}$ transfection, cells were used for subsequent experimentation.

Dual-luciferase reporter assay. HepG2 cells were co-transfected with pGL3-AdipoR2-3'UTR together with an RNA oligonucleotide. Equal amount of renilla luciferase were co-expressed as the internal control. The cell extracts were prepared $24 \mathrm{~h}$ after transfection and the luciferase activity was measured using the dual-luciferase reporter assay system (Promega Corporation). The firefly luciferase activity was normalized to Renilla luciferse activity (15).

Immunoblotting. The cells were washed twice with ice-cold phosphate-buffered saline (PBS) and lysed in ice-cold lysis buffer (100 mM Tris-HCl, pH 7.5; $300 \mathrm{mM} \mathrm{NaCl}, 1 \%$ NP-40 and $1 \mathrm{mM}$ DTT) containing protease inhibitor cocktail tablets (Cell Signaling Technology, Beverly, MA, USA). The cell lysates were resuspended in SDS loading buffer,
A

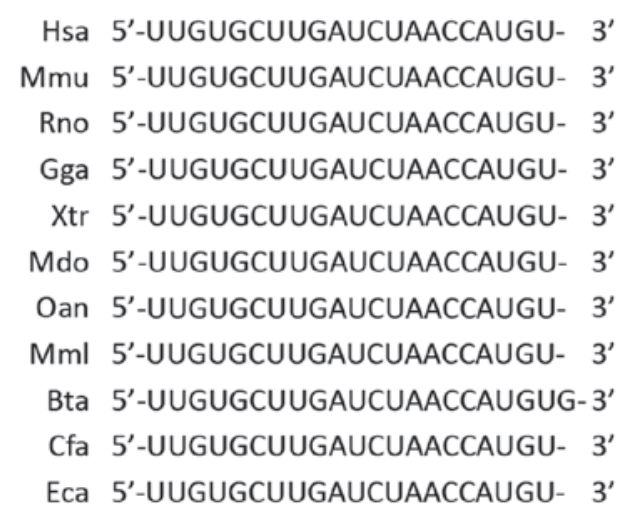

B

\begin{tabular}{|c|c|}
\hline Hsa & $\begin{array}{l}\text { 3'-UGUACCAAUCUAGUUCGUGUU-5' } \\
\text { III I I I I I } \\
\text {...GGAUCUGGUUCAACAUAGCACAAA... }\end{array}$ \\
\hline Ptr & ...GGAUCUGGUUCAACAUAGCACAAA... \\
\hline Mml & ...GGAUCUGGUUCAACAUAGCA \\
\hline Tbe & ...GAAUCUGGUUCAACAUAGCACAAC \\
\hline Иms & ...GACUCUGGUUCAACAUAGCACAAA \\
\hline Rno & ...GACUCUGGUUCAACAUAGCACAAA... \\
\hline Cpo & ...GAAUCUGGUUCAAC-- AGCACAAA... \\
\hline Ocu & ...GAAGCUGGUUCAACAUAGCACAAA... \\
\hline Sa & ...GAACCUGGUUCAACAUAGCACAAA. \\
\hline $\mathrm{E}$ & ...GAACCUGGUUUAAC-- AGCACAAA \\
\hline & ...GAAUCUGGUUCAACAUAGCACAA \\
\hline Fca & ...GAAUCUGGUUCAAUAUAGCACAA \\
\hline
\end{tabular}

Figure 1. Conservation of miR-218 and its predicted binding site within AdipoR1 3' untranslated region. (A) Evolutionary conservation of miR-218. (B) Predicted binding site of miR-218 within the AdipoR2 3' untranslated region of humans and other species. miR, microRNA; AdipoR, adiponectin receptor. Hsa, Homo sapiens; Mmu, Mus musculus; Rno, Rattus norvegicus; Gga, Gallus gallus; Xtr, Xenopus tropicalis; Mdo, Monodelphis domestica; Oan, Ornithorhynchus anatinus; Mml, Macaca mulatta; Bta, Bos taurus; Cfa, Canis familiaris; Eca, Equus caballus; Ptr, Pan troglodytes; Tbe, Tupaia belangeri; Cpo, Cavia porcellus; Ocu, Oryctolagus cuniculus; Sar, Sorex araneus; Eeu, Erinaceus europaeus; Fca, Felis catus.

heated at $95^{\circ} \mathrm{C}$ for $5 \mathrm{~min}$ and separated using SDS-PAGE. Immunocomplexes were analyzed via immunoblotting using a polyclonal goat anti-AdipoR2 antibody (sc-46751; 1:1,000; Cell Signaling Technology), followed by a polyclonal donkey anti-goat immunoglobulin $\mathrm{G}$ conjugated with horseradish peroxidase (sc-2020; 1:1,000; Santa Cruz Biotechnology, Inc., Santa Cruz, CA, USA). Immunodetection was performed with an enhanced chemiluminescence kit (Cell Signaling Technology).

Glucose uptake assay. HepG2 cells cultured on 96-well black plates were transfected with miR-218 or negative control siRNA. At $48 \mathrm{~h}$ after transfection, HepG2 cells were washed with DMEM three times and incubated with or without $10 \mu \mathrm{g} / \mathrm{ml}$ recombinant adiponectin (R\&D Systems, Inc. Minneapolis, MN, USA) for $30 \mathrm{~min}$. Following adiponectin incubation, cells were incubated with $50 \mu \mathrm{M}$ 2-(N-(7-Nitrobenz-2-oxa-1,3-diazol-4-yl) 
A

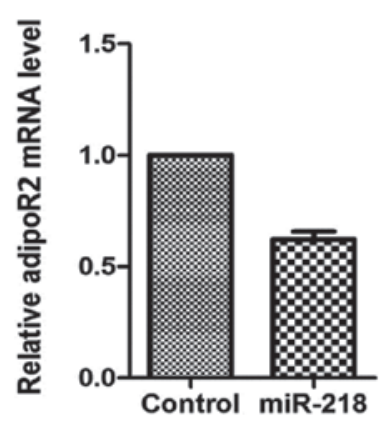

B

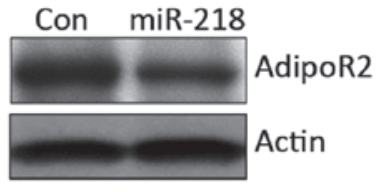

C

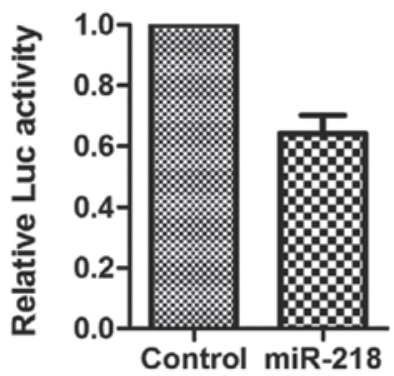

Figure 2. miR-218 downregulates AdipoR2 expression in HepG2 cells. (A) HepG2 cells were transfected with or without miR-218 and the mRNA level of AdipoR2 was detected using reverse transcription-quantitative polymerase chain reaction. (B) HepG2 cells were transfected with or without miR-218 and the protein level of AdipoR2 was detected using immunoblotting. (C) HepG2 cells were transfected with AdipoR2-untranslated region-Luciferase, together with miR-218. Luciferase activity was detected $24 \mathrm{~h}$ later. miR, microRNA; AdipoR, adiponectin receptor; Con, control.

Control miR-218
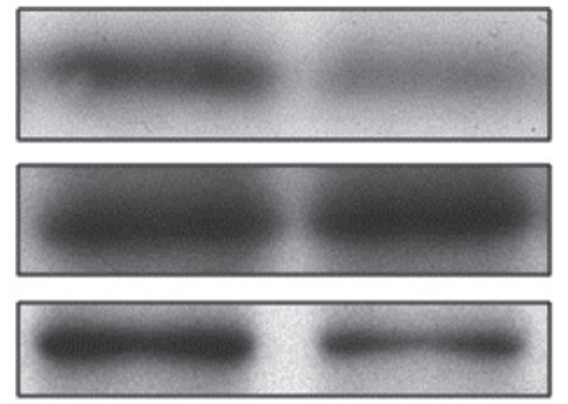

\section{p-p38MAPK}

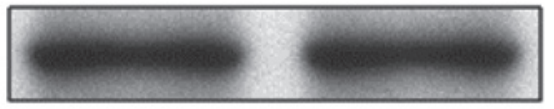

p38MAPK

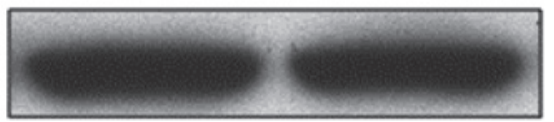

Actin

Figure 3. miR-218 inhibits AMPK and p38 MAPK pathways. HepG2 cells transfected with miR-218 or negative control were treated with or without $10 \mu \mathrm{g} / \mathrm{ml}$ adiponectin for $20 \mathrm{~min}$. Phosphorylation of p38 MAPK and AMPK and the protein levels of total p38 MAPK, AMPK and actin were detected using immunoblotting with indicated antibodies. miR, microRNA; AMPK, AMP-activated protein kinase; MAPK, mitogen-activated protein kinase.

amino)-2-deoxyglucose (2-NBDG; Invitrogen Life Technologies) in PBS for $15 \mathrm{~min}$ and then washed with additional PBS to remove excess $2-\mathrm{NBDG}$. The levels of fluorescence in the cells were measured at an excitation wavelength of $485 \mathrm{~nm}$ and an emission wavelength of $535 \mathrm{~nm}$ with Tecan infinite M200 (Tecan Group Ltd., Seestrasse, Switzerland). The fluorescence of the control group transfected with negative control and not treated with adiponectin was normalized to 1.0. The fluorescence of other groups was compared with the control group.

Statistical analysis. The data are presented as the mean \pm standard deviation. Statistical differences between groups were determined using Student's t-test. All statistical analyses were performed using Microsoft Excel 2010 (Microsoft Corp., Redmond, WA, USA). $\mathrm{P}<0.01$ was considered to indicate a statistically significant difference.

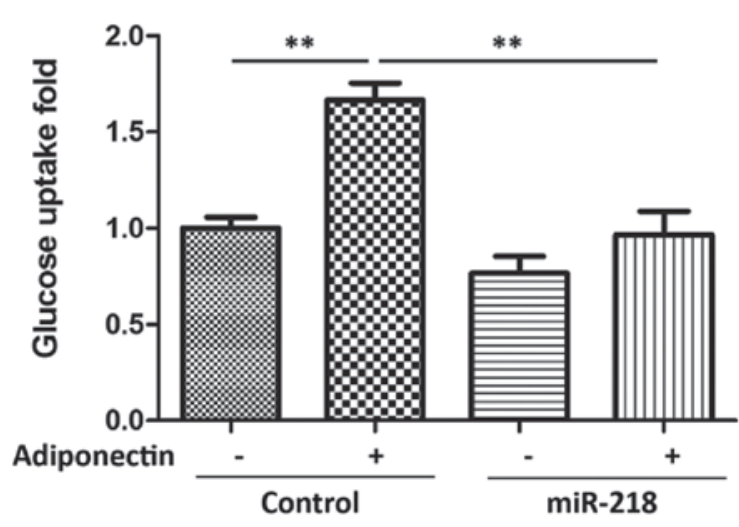

Figure 4. miR-218 inhibits adiponectin-stimulated glucose uptake. HepG2 cells transfected with miR-218 or negative control were treated with or without $10 \mu \mathrm{g} / \mathrm{ml}$ adiponectin for $20 \mathrm{~min}$. Cells were incubated with $50 \mu \mathrm{M}$ 2-(N-(7-Nitrobenz-2-oxa-1,3-diazol-4-yl) amino)-2-deoxyglucose for $15 \mathrm{~min}$ and the fluorescence was measured. Error bars represent the mean \pm standard deviation from four independent experiments. ${ }^{* *} \mathrm{P}<0.01$. miR, microRNA.

\section{Results}

miR-218 targets AdipoR2 mRNA. To identify potential AdipoR2-targeting miRNAs, an in silico search was initially performed using publicly available algorithms, including TargetScan (www.targetscan.org) and miRanda (www. microrna.org). miR-218 was identified as a candidate miRNA for targeting of AdipoR2. Phylogenetic analysis revealed that miR-218 is well-conserved among different species, ranging from Canis familiaris to Homo sapiens (Fig. 1A). A total of seven nucleotides in the 3'UTR of the human AdipoR2 mRNA are perfectly complementary to nucleotides 1-7 of miR-218 and this sequence is highly conserved in mRNA orthologs of different species (Fig. 1B).

Several approaches were subsequently employed to determine whether miR-218 regulates AdipoR2 expression. Overexpression of miR-218 mimics reduced the AdipoR2 mRNA level to $\sim 60 \%$ that of the control group (Fig. 2A). The AdipoR 2 protein level was also significantly reduced when cells were transfected with miR-218 mimics (Fig. 2B). Luciferase assays were then performed to further confirm 
the regulatory role of miR-218 in the expression of AdipoR2. The 3'UTR of the AdipoR2 gene was cloned downstream of the coding sequence of luciferase. This construct was co-transfected into HepG2 cells with miR-218 or control. The data demonstrated that miR-218 specifically decreased the luciferase levels (Fig. 2C). These results suggested that miR-218 directly binds to the 3'UTR of AdipoR 2 mRNA and reduces AdipoR2 expression.

miR-218 inhibits AMPK and p38 MAPK pathways. AdipoR2 has been revealed to be involved in the adiponectin-activated AMP-activated protein kinase and p38 mitogen-activated protein kinase pathways (7), and further mediates the effect of adiponectin on glucose metabolism. The inhibition of AdipoR 2 by miR-218 prompted the hypothesis that miR-218 may affect AMPK and p38 MAPK activation. Therefore, cells were transfected with miR-218 or negative control and cells were treated with adiponectin. The data revealed that phosphorylation of p38 MAPK and AMPK decreased in miR-218 expressing cells (Fig. 3), suggesting that miR-218 inhibited the adiponectin-activated AMPK and p38 MAPK pathways.

miR-218 inhibits adiponecin-induced glucose uptake. Adiponectin enhances glucose uptake, therefore, it was proposed that miR-218 may regulate adiponectin-induced glucose metabolism. To assess this hypothesis, HepG2 cells transfected with miR-218 were then incubated with adiponectin and the uptake of 2-NBDG, a metabolizable fluorescent derivative of glucose, was measured. As expected, adiponectin treatment was observed to markedly increase glucose uptake by $\sim 1.67 \pm 0.15$ fold more than that of the untreated group. Adiponectin-induced glucose uptake was reduced by $0.97 \pm 0.21$ fold when miR-218 was expressed (Fig. 4), indicating that miR-218 inhibits adiponectin-induced glucose uptake.

\section{Discussion}

As adiponectin has been observed to exhibit a significant antidiabetic function, elucidating the adiponectin signaling pathway is essential to harness the therapeutic potential of this hormone. Adiponectin receptors may have important roles in adiponectin signaling transduction. However, the mechanism regulating the expression of adiponectin receptors remains to be elucidated. In the present study, it was demonstrated that miR-218 directly targets AdipoR2. In addition, it was demonstrated that miR-218 inhibits adiponectin signaling and glucose uptake by repressing AdipoR2 expression.

The expression levels of AdipoR1 and AdipoR2 are closely associated with insulin sensitivity. AdipoR1 expression was decreased in the skeletal muscle of Type 2 diabetic patients (16). The expression of AdipoR1 and AdipoR2 in skeletal muscle and the liver is significantly increased in fasted mice and decreased in refed mice (17). Insulin deficiency induced by streptozotocin increased the expression of AdipoR1 and AdipoR2, and insulin replenishment reduced the expression of AdipoR1 and AdipoR2 in vivo (17). Insulin also reduced the expression of AdipoR1 and AdipoR2 in hepatocytes or myocytes via the phosphoinositide 3-kinase/ Foxo1-dependent pathway in vitro (17). However, other studies have demonstrated that insulin may also decrease the expression of AdipoR1, but not AdipoR2 in primary cultured neonatal rat ventricular myocytes (18) and L6 myoblasts (19). The inconsistency in the expression of AdipoRs suggested that the expression regulation of AdipoR1 and AdipoR2 is complicated and not restricted to the transcriptional level. The present data indicated that miR-218 regulates AdipoR2 expression at the posttranscriptional level, nevertheless the physiological and pathological significance of miR-218-repressed AdipoR2 expression requires further investigation.

It has been observed that miR-218 is a multifunctional microRNA. It acts as a tumor suppressor in numerous types of cancer, including oral cancer (20), mesenchymal glioblastoma (21) and medulloblastoma (22). miR-218 inhibits the expression of the mammalian target of rapamycin component Rictor and therefore inhibits AKT phosphorylation and cell proliferation (20). Through targeting the receptor tyrosine kinase signaling pathway, miR-218 inhibits the activation of hypoxia-inducible factor and thereby inhibits glioblastoma multiforme cell survival and angiogenesis (21). In addition to its tumor-suppressing effects, miR-218 was also observed to be involved in cell differentiation. Through downregulation of Wnt signaling inhibitors, miR-218 may activate Wnt signaling to promote the differentiation of osteoblasts (23) or osteogenic differentiation of human adipose tissue-derived stem cells (24). In the present study, the role of miR-218 in glucose metabolism was revealed, for the first time to the best of our knowledge, as demonstrated by the observation that overexpression of miR-218 inhibits the AMPK and p38 MAPK pathways (Fig. 3) and adiponectin-induced glucose uptake (Fig. 4). Further studies using mouse models may assist in elucidating the function of miR-218 in glucose metabolism and the development of diabetes.

In conclusion, AdipoR 2 has been identified as a direct target of miR-218. Furthermore, miR-218 was revealed to inhibit adiponectin-induced AMPK and p38 MAPK activation and glucose uptake in HepG 2 cells. These results indicated that miR-218 targets AdipoR2 to inhibit the adiponectin signaling pathway. This data may also provide a potential target for diabetes therapy through inhibiting miR-218.

\section{Acknowledgements}

The present study was supported by grants from the Natural Science Foundation of Zhejiang Province (grant nos. Y14H040003 and Y14H040004), the Medical Foundation of Zhejiang Province (grant no. 2013KYA207), and the Shaoxing Science and Technology Bureau Program (grant nos. 2013B70079 and 2013B70060). Dr Tao Zhang was awarded a grant by the China Postdoctoral Science Foundation.

\section{References}

1. Gil-Campos M, Cañete RR and Gil A: Adiponectin, the missing link in insulin resistance and obesity. Clin Nutr 23: 963-974, 2004.

2. Ukkola O and Santaniemi M: Adiponectin: a link between excess adiposity and associated comorbidities? J Mol Med (Berl) 80: 696-702, 2002.

3. Yamauchi T, Kamon J, Waki H, et al: The fat-derived hormone adiponectin reverses insulin resistance associated with both lipoatrophy and obesity. Nat Med 7: 941-946, 2001. 
4. Kadowaki T, Yamauchi T, Kubota N, et al: Adiponectin and adiponectin receptors in insulin resistance, diabetes, and the metabolic syndrome. J Clin Invest 116: 1784-1792, 2006.

5. Maeda N, Shimomura I, Kishida K, et al: Diet-induced insulin resistance in mice lacking adiponectin/ACRP30. Nat Med 8: 731-737, 2002.

6. Li S, Shin HJ, Ding EL, et al: Adiponectin levels and risk of type 2 diabetes: a systematic review and meta-analysis. JAMA 302 : $179-188,2009$.

7. Yamauchi T and Kadowaki T: Adiponectin receptor as a key player in healthy longevity and obesity-related diseases. Cell Metab 17: 185-196, 2013.

8. Bartel DP: MicroRNAs: genomics, biogenesis, mechanism, and function. Cell 116: 281-297, 2004.

9. Fernández-Hernando C, Ramírez CM, Goedeke L and Suárez Y: MicroRNAs in metabolic disease. Arterioscler Thromb Vasc Biol 33: 178-185, 2013.

10. Poy MN, Eliasson L, Krutzfeldt J, et al: A pancreatic islet-specific microRNA regulates insulin secretion. Nature 432: 226-230, 2004.

11. Frost RJ and Olson EN: Control of glucose homeostasis and insulin sensitivity by the Let-7 family of microRNAs. Proc Nat Acad Sci USA 108: 21075-21080, 2011.

12. Zhu H, Shyh-Chang N, Segrè AV, et al: The Lin28/let-7 axis regulates glucose metabolism. Cell 147: 81-94, 2011.

13. Trajkovski M, Hausser J, Soutschek J, et al: MicroRNAs 103 and 107 regulate insulin sensitivity. Nature 474: 649-653, 2011.

14. Pullen TJ, da Silva Xavier G, Kelsey G and Rutter GA: miR-29a and miR-29b contribute to pancreatic beta-cell-specific silencing of monocarboxylate transporter 1 (Mct1). Mol Cell Biol 31: 3182-3194, 2011.

15. Gao X, Dong H, Lin C, et al: Reduction of AUF1-mediated follistatin mRNA decay during glucose starvation protects cells from apoptosis. Nucleic Acids Res 42: 10720-10730, 2014.
16. Debard C, Laville M, Berbe V, et al: Expression of key genes of fatty acid oxidation, including adiponectin receptors, in skeletal muscle of Type 2 diabetic patients. Diabetologia 47: 917-925, 2004

17. Tsuchida A, Yamauchi T, Ito Y, et al: Insulin/Foxo1 pathway regulates expression levels of adiponectin receptors and adiponectin sensitivity. J Biol Chem 279: 30817-30822, 2004.

18. Cui XB, Wang C, Li L, et al: Insulin decreases myocardial adiponectin receptor 1 expression via PI3K/Akt and FoxO1 pathway. Cardiovasc Res 93: 69-78, 2012.

19. Fang X, Palanivel R, Zhou X, et al: Hyperglycemia- and hyperinsulinemia-induced alteration of adiponectin receptor expression and adiponectin effects in L6 myoblasts. J Mol Endocrinol 35: 465-476, 2005

20. Uesugi A, Kozaki K, Tsuruta T, et al: The tumor suppressive microRNA miR-218 targets the mTOR component Rictor and inhibits AKT phosphorylation in oral cancer. Cancer Res 71: 5765-5778, 2011

21. Mathew LK, Skuli N, Mucaj V, et al: mir-218 opposes a critical RTK-HIF pathway in mesenchymal glioblastoma. Proc Natl Acad Sci USA 111: 291-296, 2014.

22. Venkataraman S, Birks DK, Balakrishnan I, et al: MicroRNA 218 acts as a tumor suppressor by targeting multiple cancer phenotype-associated genes in medulloblastoma. J Biol Chem 288: 1918-1928, 2013.

23. Hassan MQ, Maeda Y, Taipaleenmaki H, et al: mir-218 directs a wnt signaling circuit to promote differentiation of osteoblasts and osteomimicry of metastatic cancer cells. J Biol Chem 287: 42084-42092, 2012

24. Zhang WB, Zhong WJ and Wang L: A signal-amplification circuit between miR-218 and Wnt/beta-catenin signal promotes human adipose tissue-derived stem cells osteogenic differentiation. Bone 58: 59-66, 2014. 